\title{
带插值的自适应网格重构算法求解带移动热源的 反应扩散方程的理论分析
}

\author{
马敬堂 ${ }^{(1)}$ ，姜英军 ${ }^{2}$ \\ (1) 西南财经大学经济数学学院, 成都 611130; \\ (2) 长沙理工大学数学与计算科学系, 长沙 410076 \\ E-mail: mjt@swufe.edu.cn, jiangyingjun@csust.edu.cn \\ 收稿日期: 2009-12-25；接受日期：2011-01-20；＊通信作者 \\ 国家自然科学基金 (批准号: 10901027) 资助项目
}

摘要 本文研究一个带插值的网格重构算法求解一类带移动热源的反应扩散方程. 算法包括两步: 第 一步是用旧时间网层上的计算解计算新时间层上的空间网格; 第二步是使用有限差分方法在新时间层 空间网格上离散方程, 并且将旧时间层上计算解的插值作为初始值. 对于时间, 我们获得了一阶收敛 结果. 对于空间, 我们证明了使用线性插值算法的一阶收玫性和使用二次插值算法的二阶收敛性. 数 值例子肯定了本文的理论结果.

关键词 移动网格方法 稳定性和收敛性 反应扩散方程

MSC (2000) 主题分类 $65 \mathrm{~N} 50,65 \mathrm{M} 50,35 \mathrm{~K} 55,35 \mathrm{~K} 57$

\section{1 引言}

本文求解下面问题

$$
u_{t}-u_{x x}=\delta\left(x-x_{0}(t)\right) f\left(x_{0}, t\right), \quad a \leqslant x \leqslant b, \quad t>0,
$$

边界条件为 $u(a, t)=0, u(b, t)=0$, 初值条件为 $u(x, 0)=u_{0}(x)$. 这里 $f$ 和 $x_{0}$ 是给定的连续函数, $\delta$ 是 Dirac delta 函数. 当使用激光沿着给定路径对某可燃物质加热时, 上述方程可模拟其热传导过程, 其 中 $u$ 是物体的温度 (见 $[1-3]$ ). $x_{0}(t)$ 即是局部热源所作用的位置. 由于上述方程的解具有奇异性且不 知道奇异解的性质和奇异点的位置, 自适应移动网格方法将是对其进行数值模拟的一类理想方法. 本 文将使用一种简单的移动网格算法对上述问题进行求解. 如早期文章所述, 移动有限差分算法分为三 类: BJCN 算法 (即 Bonnerot-Jamet Crank-Nicolson 算法, 可以看作是 Godunov 算法的一种), IEL 算 法 (即 Implicit-Euler Lagrangian 算法), 网格重构算法 (即 Remeshing Scheme). BJCN 算法最先在文献 [4] 中提出. Jamet ${ }^{[5]}$ 用此方法求解带移动边界的热传导方程, 并对其收玫性进行了证明, 文中在每一 个时刻都使用一致网格, 且证明不能脱离网格的一致性. 近来, $\mathrm{Ma}^{[6]}$ 给出了一个对非一致网格更一般 性的证明. Mackenzie 和 Mekwi ${ }^{[7]}$ 对守恒 IEL 算法 (亦称 Arbitrary Lagrangian-Eulerian (ALE) 算法) 给出了一个渐近二阶收玫性证明. 这个结论最近又被 $\mathrm{Ma}$ 等 ${ }^{[8]}$ 推广到偏积分微分方程. Mackenzie 和 Madzvamuse 在文献 [9] 中研究了使用守恒和非守恒 IEL 算法在指数增长区域求解反应扩散方程的稳 
定性和收敛性. 网格重构算法又称为 “Adaptive Mesh Redistribution Algorithms”, 最先由 Blom 等 [10]

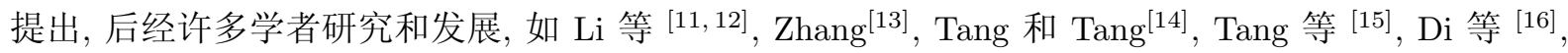
Zhang 和 Tang ${ }^{[17]}$. 如文章 $[11,12,14-16]$ 中所述, 网格重构算法在高维问题中较容易使用. 正因如此, 我们将研究这种方法的收玫性理论. 在本文中, 对问题 (1) 我们研究一种带插值的网格重构算法, 分析 算法的稳定性和收玫性. 在文献 $[18,19]$ 中, 也对这种网格重构算法做了描述和应用.

接下来, 我们介绍网格重构算法求解问题 (1) 的过程. 给定时间网格

$$
0 \equiv t_{0}<t_{1}<\cdots<t_{n}<\cdots
$$

其中 $\Delta t_{n}=t_{n+1}-t_{n}$. 在时刻 $t_{n}, n=0,1, \ldots$, 定义与时间相关的空间网格

$$
a=x_{0}^{n}<x_{1}^{n}<\cdots<x_{N}^{n}=b .
$$

令 $h_{j}^{n}=x_{j}^{n}-x_{j-1}^{n}, u_{j}^{n+1}$ 表示 $u\left(x_{j}^{n+1}, t_{n+1}\right)$ 的逼近值. 自适应网格重构算法定义为:

$$
\frac{u_{j}^{n+1}-\widetilde{u}_{j}^{n}}{\Delta t_{n}}-\frac{\frac{u_{j+1}^{n+1}-u_{j}^{n+1}}{x_{j+1}^{n+1}-x_{j}^{n+1}}-\frac{u_{j}^{n+1}-u_{j-1}^{n+1}}{x_{j}^{n+1}-x_{j-1}^{n+1}}}{\frac{x_{j+1}^{n+1}-x_{j-1}^{n+1}}{2}}=(\text { Term })_{j}^{n+1},
$$

其中 $\widetilde{u}_{j}^{n}$ 由线性插值

$$
\widetilde{u}_{j}^{n}=\Pi\left(u^{n} ; x_{j}^{n}, x_{j+1}^{n}\right)\left(x_{j}^{n+1}\right)=\frac{x_{j+1}^{n}-x_{j}^{n+1}}{x_{j+1}^{n}-x_{j}^{n}} u_{j}^{n}+\frac{x_{j}^{n+1}-x_{j}^{n}}{x_{j+1}^{n}-x_{j}^{n}} u_{j+1}^{n}
$$

或二次插值

$$
\begin{aligned}
\widetilde{u}_{j}^{n}= & \Pi\left(u^{n} ; x_{j-1}^{n}, x_{j}^{n}, x_{j+1}^{n}\right)\left(x_{j}^{n+1}\right) \\
= & \frac{\left(x_{j}^{n+1}-x_{j}^{n}\right)\left(x_{j}^{n+1}-x_{j+1}^{n}\right)}{\left(x_{j-1}^{n}-x_{j}^{n}\right)\left(x_{j-1}^{n}-x_{j+1}^{n}\right)} u_{j-1}^{n} \\
& +\frac{\left(x_{j}^{n+1}-x_{j-1}^{n}\right)\left(x_{j}^{n+1}-x_{j+1}^{n}\right)}{\left(x_{j}^{n}-x_{j-1}^{n}\right)\left(x_{j}^{n}-x_{j+1}^{n}\right)} u_{j}^{n}+\frac{\left(x_{j}^{n+1}-x_{j}^{n}\right)\left(x_{j}^{n+1}-x_{j-1}^{n}\right)}{\left(x_{j+1}^{n}-x_{j}^{n}\right)\left(x_{j+1}^{n}-x_{j-1}^{n}\right)} u_{j+1}^{n}
\end{aligned}
$$

给出, 这里 $(\mathrm{Term})_{j}^{n+1}$ 可以看做是移动热源的逼近, 具体形式将由后面式子 (16) 给出.

在以往的文献中, 一般需要使用下面条件来证明移动网格算法的收敛性 (参见 $[5,7,8,20]$ ):

(C1) 网格密度比条件

$$
1-C \Delta t_{n} \leqslant \frac{x_{j}^{n+1}-x_{j-1}^{n+1}}{x_{j}^{n}-x_{j-1}^{n}} \leqslant 1+C \Delta t_{n}
$$

(C2) 网格移动速度条件

$$
\frac{\left|x_{j}^{n+1}-x_{j}^{n}\right|}{\Delta t_{n}} \leqslant C
$$

(C3) 网格的光滑性条件

$$
\left|\left(x_{j+2}^{n}-x_{j+1}^{n}\right)-\left(x_{j+1}^{n}-x_{j}^{n}\right)\right| \leqslant C \min \left(\left(x_{j+2}^{n}-x_{j+1}^{n}\right)^{2},\left(x_{j+1}^{n}-x_{j}^{n}\right)^{2}\right) ;
$$

(C4) CFL 条件

$$
\Delta t_{n} \leqslant C\left(x_{j}^{n}-x_{j-1}^{n}\right)^{2} .
$$




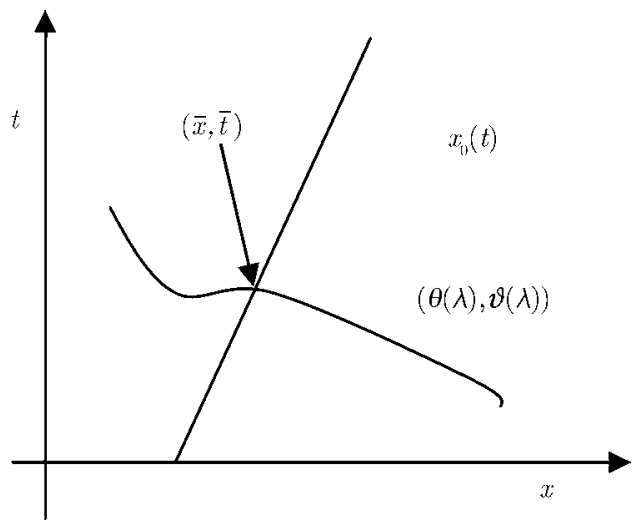

图 1 跳跃概念图示

本文中我们将在条件 (C1)-(C4) 的前提下, 证明自适应网格重构算法 (2) 在使用线性插值时具有一阶 收敛性, 在使用二次插值时具有二阶收敛性.

本文内容组织如下: 第 2 节, 我们推导截断误差和一些有用的结论; 第 3 节, 我们将证明算法在使 用线性插值时的收玫阶; 第 4 节, 我们将证明算法在使用二次插值时的收敛阶; 第 5 节, 我们给出几个 数值算例来验证所得的理论分析结果; 最后, 我们对本文进行总结.

在本文中, 所有 $C$ 均表示与时间和空间网格无关的正常数.

\section{2 截断误差估计和两个有用的引理}

由于问题 (1) 的解在移动曲线 $x_{0}(t)$ 处具有奇异性, 导数解的逼近需要特殊处理才能使整个算法 达到最优精度. Ma 和 Jiang 在文献 [20] 中, 结合解在 $x_{0}(t)$ 处的跳跃信息 (可以计算出来), 引入一个 辅助函数, 得到各种导数的有效逼近, 进而得到具有最优收玫阶的算法. 接下来, 我们将使用文献 [20] 中有关的数学符号, 并对跳跃进行计算, 从而得到对导数解的有效逼近.

假定 $\varphi(x, t)$ 在 $x_{0}(t)$ 两侧连续且有界. 取任意给定光滑路径 $(\theta(\lambda), \vartheta(\lambda))$, 使其在 $\lambda$ 增加时从左向 右经点 $(\bar{x}, \bar{t})$ 穿过 $x_{0}(t),(\theta(\bar{\lambda}), \vartheta(\bar{\lambda}))=(\bar{x}, \bar{t})$, 我们定义 $\varphi$ 在 $(\bar{x}, \bar{t})$ 处的跳跃为 (见图 1$)$ :

$$
[\varphi]_{(\bar{x}, \bar{t})}=\lim _{\varepsilon \rightarrow 0^{+}}(\varphi(\theta(\bar{\lambda}+\epsilon), \vartheta(\bar{\lambda}+\epsilon))-\varphi(\theta(\bar{\lambda}-\epsilon), \vartheta(\bar{\lambda}-\epsilon))) .
$$

对于一个一元函数 $g(x)$, 我们定义当 $x$ 经过 $\xi$ 时跳跃为

$$
{ }_{x}[g]_{\xi}=\lim _{\varepsilon \rightarrow 0^{+}}(g(\xi+\epsilon)-g(\xi-\epsilon)) .
$$

由跳跃的定义可知

$$
{ }_{x}[\varphi(\cdot, \bar{t})]_{\bar{x}}=[\varphi]_{(\bar{x}, \bar{t})}
$$

和

$$
{ }_{t}[\varphi(\bar{x}, \cdot)]_{\bar{t}}=\sigma[\varphi]_{(\bar{x}, \bar{t})},
$$

见图 2, 其中左侧图对应 $\sigma=1$ 情形, 右侧图对应 $\sigma=-1$ 情形.

$u, u_{t}, u_{x}$ 在 $x=x_{0}(t)$ 的跳跃可以先计算出来. 由于原方程 (1) 在 $x \neq x_{0}$ 的区域是热传导方程, 所以解 $u$ 在整个区域是连续函数, 且在 $x \neq x_{0}$ 的区域是光滑的. 在点 $\left(x_{0}(t), t\right)$ 处, 这些函数的跳跃分 

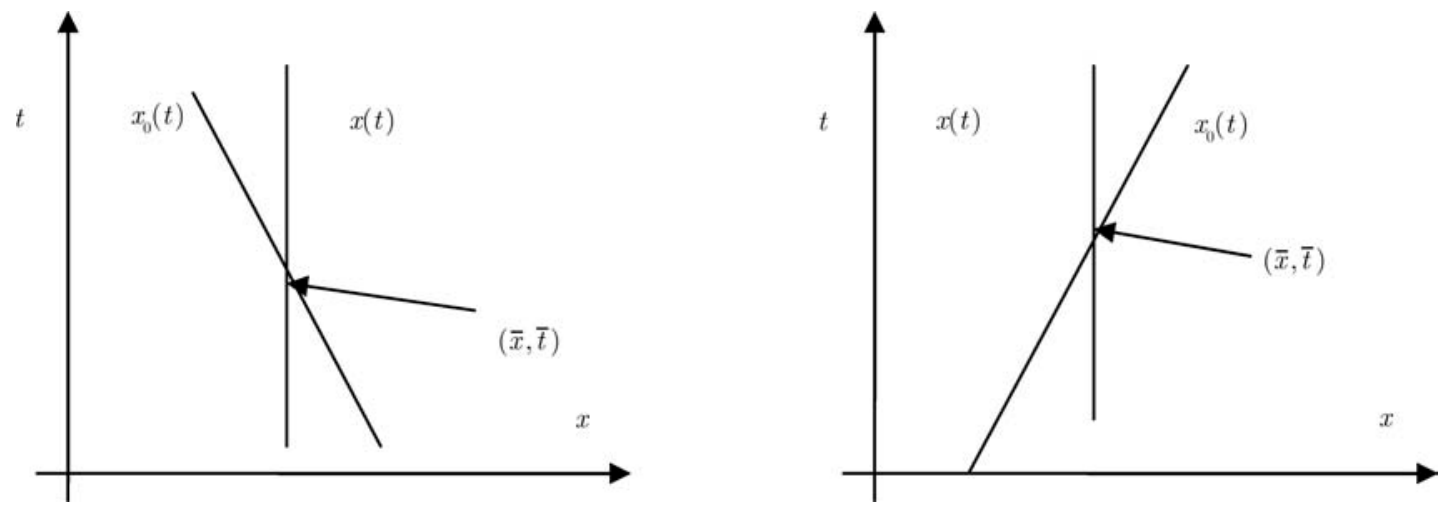

图 2 左侧图形对应 $\sigma=1$, 右侧图形对应 $\sigma=-1$

别为:

$$
[u]_{\left(x_{0}(t), t\right)}=0, \quad\left[u_{x}\right]_{\left(x_{0}(t), t\right)}=-f\left(x_{0}(t), t\right) .
$$

解 $u(x, t)$ 沿着方向 $\left(x_{0}^{\prime}(t), 1\right)$ 的跳跃为零, 即

$$
\left[u_{x}\right]_{\left(x_{0}(t), t\right)} x_{0}^{\prime}(t)+\left[u_{t}\right]_{\left(x_{0}(t), t\right)}=0 .
$$

由此可知

$$
\left[u_{t}\right]_{\left(x_{0}(t), t\right)}=-x_{0}^{\prime}(t)\left[u_{x}\right]_{\left(x_{0}(t), t\right)} .
$$

结合 (1) 和 (9), 我们得到

$$
\left[u_{x x}\right]_{\left(x_{0}(t), t\right)}=\left[u_{t}\right]_{\left(x_{0}(t), t\right)}=x_{0}^{\prime}(t) f\left(x_{0}(t), t\right) .
$$

如文献 [20] 所述, 项 $u_{x x}\left(x_{j}^{n+1}+, t_{n+1}\right)$ 和 $u_{t}\left(x_{j}^{n+1}+, t_{n+1}\right)$ 可以展开成如下形式:

$$
\begin{aligned}
& u_{x x}\left(x_{j}^{n+1}+, t_{n+1}\right)=\frac{\frac{u\left(x_{j+1}^{n+1}, t_{n+1}\right)-u\left(x_{j}^{n+1}, t_{n+1}\right)}{h_{j+1}^{n+1}}-\frac{u\left(x_{j}^{n+1}, t_{n+1}\right)-u\left(x_{j-1}^{n+1}, t_{n+1}\right)}{h_{j}^{n+1}}}{\frac{h_{j}^{n+1}+h_{j+1}^{n+1}}{2}}+(\text { Term- } 1)_{j}^{n+1}+\cdots, \\
& u_{t}\left(x_{j}^{n+1}+, t_{n+1}\right)=\frac{u\left(x_{j}^{n+1}, t_{n+1}\right)-u\left(x_{j}^{n+1}, t_{n}\right)}{t_{n+1}-t_{n}}+(\text { Term- } 2)_{j}^{n+1}+\cdots,
\end{aligned}
$$

其中 $(\text { Term- } 1)_{j}^{n+1},(\text { Term- } 2)_{j}^{n+1}$ 定义为

$$
(\text { Term- } 1)_{j}^{n+1}=\left\{\begin{array}{l}
0, \quad \text { 若 } x_{0}\left(t_{n+1}\right) \notin\left(x_{j-1}^{n+1}, x_{j+1}^{n+1}\right) ; \\
\frac{2}{h_{j}^{n+1}+h_{j+1}^{n+1}}\left(-\frac{h_{j, 1}^{n+1}}{h_{j}^{n+1}}\left[u_{x}\right]_{\left(x_{0}\left(t_{n+1}\right), t_{n+1}\right)}+\frac{\left(h_{j, 1}^{n+1}\right)^{2}}{2 h_{j}^{n+1}}\left[u_{x x}\right]_{\left(x_{0}\left(t_{n+1}\right), t_{n+1}\right)}\right), \\
\quad \text { 若 } x_{0}\left(t_{n+1}\right) \in\left(x_{j-1}^{n+1}, x_{j}^{n+1}\right] ; \\
-\frac{2}{h_{j}^{n+1}+h_{j+1}^{n+1}}\left(\frac{h_{j, 2}^{n+1}}{h_{j+1}^{n+1}}\left[u_{x}\right]_{\left(x_{0}\left(t_{n+1}\right), t_{n+1}\right)}+\frac{\left(h_{j, 2}^{n+1}\right)^{2}}{2 h_{j+1}^{n+1}}\left[u_{x x}\right]_{\left(x_{0}\left(t_{n+1}\right), t_{n+1}\right)}\right), \\
\quad \text { 若 } x_{0}\left(t_{n+1}\right) \in\left(x_{j}^{n+1}, x_{j+1}^{n+1}\right),
\end{array}\right.
$$


和

$$
(\mathrm{Term}-2)_{j}^{n+1}= \begin{cases}\frac{\bar{t}-t_{n}}{t_{n+1}-t_{n}}{ }_{t}\left[u_{t}\right]_{\bar{t}}, & \text { 若 } x_{0}(t) \text { 交于 } L_{j}^{n+1} \text { 及 } x_{0}^{\prime}(\bar{t})<0, \\ 0, & \text { 否则, }\end{cases}
$$

这里 $h_{j, 1}^{n+1}=x_{0}\left(t_{n+1}\right)-x_{j-1}^{n+1}, h_{j, 2}^{n+1}=x_{j+1}^{n+1}-x_{0}\left(t_{n+1}\right), L_{j}^{n+1}$ 表示连接 $\left(x_{j}^{n+1}, t_{n}\right)$ 和 $\left(x_{j}^{n+1}, t_{n+1}\right)$ 的 线段 (不包含点 $\left(x_{j}^{n}, t_{n}\right)$ ), 并且 $x_{0}(t)$ 与 $L_{j}^{n+1}$ 的交点对应的参数为 $t=\bar{t}$. 注意, 由于 (9) 和 (11), 故 $(\text { Term- } 1)_{j}^{n+1}$ 和 $(\text { Term- } 2)_{j}^{n+1}$ 是已知的.

在以上各种逼近形式基础上, 算法 (2) 中的项 $(\mathrm{Term})_{j}^{n+1}$ 定义为

$$
(\text { Term })_{j}^{n+1}=(\text { Term- } 1)_{j}^{n+1}-(\text { Term- } 2)_{j}^{n+1} .
$$

通过下述方程, 我们给出算法 $(2)$ 在 $\left(x_{j}^{n+1}+, t_{n+1}\right)$ 点处的截断误差的定义, 记其为 $\tau_{j}^{n+1}$, 于是

$$
\begin{aligned}
& \frac{u\left(x_{j}^{n+1}, t_{n+1}\right)-u\left(x_{j}^{n+1}, t_{n}\right)}{\Delta t_{n}} \\
& =\frac{2}{x_{j+1}^{n+1}-x_{j-1}^{n+1}}\left[\frac{u\left(x_{j+1}^{n+1}, t_{n+1}\right)-u\left(x_{j}^{n+1}, t_{n+1}\right)}{x_{j+1}^{n+1}-x_{j}^{n+1}}-\frac{u\left(x_{j}^{n+1}, t_{n+1}\right)-u\left(x_{j-1}^{n+1}, t_{n+1}\right)}{x_{j}^{n+1}-x_{j-1}^{n+1}}\right] \\
& \quad+(\text { Term })_{j}^{n+1}+\tau_{j}^{n+1} .
\end{aligned}
$$

定理 1 (截断误差估计) 由 (17) 所定义的截断误差满足

$$
\left|\tau_{j}^{n+1}\right| \leqslant C \begin{cases}\max \left\{h_{j+1}^{n+1}-h_{j}^{n+1},\left(h_{j+1}^{n+1}\right)^{2}+\left(h_{j}^{n+1}\right)^{2}, \Delta t_{n}\right\}, & \text { 若 } x_{0}\left(t_{n+1}\right) \notin\left(x_{j-1}^{n+1}, x_{j+1}^{n+1}\right) ; \\ \max \left\{h_{j+1}^{n+1}+h_{j}^{n+1}, \Delta t_{n}\right\}, & \text { 若 } x_{0}\left(t_{n+1}\right) \in\left(x_{j-1}^{n+1}, x_{j+1}^{n+1}\right) .\end{cases}
$$

证明 定理证明参见 [20].

本节最后, 我们引入两个引理, 这两个引理将在后面分析中使用.

引理 1 若条件 (C3) 成立, 对任意指标 $j$ 和 $n$, 我们有如下不等式:

$$
x_{j+1}^{n}-x_{j-1}^{n} \leqslant C \min \left(\left(x_{j+2}^{n}-x_{j}^{n}\right),\left(x_{j}^{n}-x_{j-2}^{n}\right)\right),
$$

和

$$
\frac{x_{j+1}^{n}-x_{j-1}^{n}}{2} \leqslant C \min \left(\left(x_{j+1}^{n}-x_{j}^{n}\right),\left(x_{j}^{n}-x_{j-1}^{n}\right)\right) .
$$

证明 我们做如下分解

$$
x_{j+1}^{n}-x_{j-1}^{n}=\left[x_{j+2}^{n}-x_{j}^{n}\right]-\left[\left(x_{j+2}^{n}-x_{j}^{n}\right)-\left(x_{j+1}^{n}-x_{j-1}^{n}\right)\right] .
$$

由 (C3), 第二项可被估计为

$$
\begin{aligned}
& \left(x_{j+2}^{n}-x_{j}^{n}\right)-\left(x_{j+1}^{n}-x_{j-1}^{n}\right) \\
& \quad=\left[\left(x_{j+2}^{n}-x_{j+1}^{n}\right)-\left(x_{j+1}^{n}-x_{j}^{n}\right)\right]+\left[\left(x_{j+1}^{n}-x_{j}^{n}\right)-\left(x_{j}^{n}-x_{j-1}^{n}\right)\right] \\
& \quad \leqslant C\left[\min \left(\left(x_{j+2}^{n}-x_{j+1}^{n}\right)^{2},\left(x_{j+1}^{n}-x_{j}^{n}\right)^{2}\right)+\min \left(\left(x_{j+1}^{n}-x_{j}^{n}\right)^{2},\left(x_{j}^{n}-x_{j-1}^{n}\right)^{2}\right)\right] \\
& \quad \leqslant C\left[\left(x_{j+2}^{n}-x_{j+1}^{n}\right)^{2}+\left(x_{j+1}^{n}-x_{j}^{n}\right)^{2}\right] \\
& \quad \leqslant C\left[\left(x_{j+2}^{n}-x_{j+1}^{n}\right)+\left(x_{j+1}^{n}-x_{j}^{n}\right)\right]^{2}
\end{aligned}
$$




$$
=C\left(x_{j+2}^{n}-x_{j}^{n}\right)^{2} .
$$

将上式代入 (20) 可得

$$
x_{j+1}^{n}-x_{j-1}^{n} \leqslant C\left(x_{j+2}^{n}-x_{j}^{n}\right) .
$$

类似地有

$$
x_{j+1}^{n}-x_{j-1}^{n}=\left[x_{j}^{n}-x_{j-2}^{n}\right]+\left[\left(x_{j+1}^{n}-x_{j-1}^{n}\right)-\left(x_{j}^{n}-x_{j-2}^{n}\right)\right],
$$

于是可得

$$
x_{j+1}^{n}-x_{j-1}^{n} \leqslant C\left(x_{j}^{n}-x_{j-2}^{n}\right) .
$$

因此不等式 (18) 得证. 接下来我们证明不等式 (19). 为此我们考虑恒等式

$$
\frac{x_{j+1}^{n}-x_{j-1}^{n}}{2}=\left(x_{j+1}^{n}-x_{j}^{n}\right)-\frac{\left(x_{j+1}^{n}-x_{j}^{n}\right)-\left(x_{j}^{n}-x_{j-1}^{n}\right)}{2} .
$$

于是应用 $(\mathrm{C} 3)$, 我们得到

$$
\frac{x_{j+1}^{n}-x_{j-1}^{n}}{2} \leqslant\left(x_{j+1}^{n}-x_{j}^{n}\right)+\frac{C}{2}\left(x_{j+1}^{n}-x_{j}^{n}\right)^{2} \leqslant C\left(x_{j+1}^{n}-x_{j}^{n}\right) .
$$

类似地, 我们用恒等式

$$
\frac{x_{j+1}^{n}-x_{j-1}^{n}}{2}=\left(x_{j}^{n}-x_{j-1}^{n}\right)+\frac{\left(x_{j+1}^{n}-x_{j}^{n}\right)-\left(x_{j}^{n}-x_{j-1}^{n}\right)}{2},
$$

及条件 (C3) 可得

$$
\frac{x_{j+1}^{n}-x_{j-1}^{n}}{2} \leqslant C\left(x_{j}^{n}-x_{j-1}^{n}\right) .
$$

因此不等式 (19) 得证.

引理 $\mathbf{2}^{[21]}$ 对任意 $1 \leqslant n \leqslant m$, 令 $\Delta t_{n}>0, \alpha_{n}, \gamma_{n}, \beta_{n}, q_{n} \geqslant 0$, 满足 $\beta_{n} \Delta t_{n} \leqslant 1 / 2, \beta=\max _{n} \beta_{n}$. 如果

$$
\left(1-\beta_{n} \Delta t_{n}\right) q_{n}+\Delta t_{n} \gamma_{n} \leqslant \Delta t_{n} \alpha_{n}+\left(1+\beta_{n} \Delta t_{n}\right) q_{n-1},
$$

则存在一个正常数 $C_{m}$ 使得

$$
\max _{0 \leqslant n \leqslant m} q_{n}+\sum_{n=1}^{m} \gamma_{n} \Delta t_{n} \leqslant C_{m}\left\{q_{0}+\sum_{n=1}^{m} \alpha_{n} \Delta t_{n}\right\},
$$

其中

$$
C_{m}=\prod_{n=1}^{m} \frac{1+\beta_{n} \Delta t_{n}}{1-\beta_{n} \Delta t_{n}} \leqslant C \exp \left(c \sum_{n=1}^{m} \beta_{n} \Delta t_{n}\right) \leqslant C \exp (c \beta T) .
$$

\section{3 线性插值情形}

定义线性插值算子:

$$
\Pi\left(u^{n} ; x_{j}^{n}, x_{j+1}^{n}\right)(x)=\frac{x_{j+1}^{n}-x}{x_{j+1}^{n}-x_{j}^{n}} u_{j}^{n}+\frac{x-x_{j}^{n}}{x_{j+1}^{n}-x_{j}^{n}} u_{j+1}^{n} .
$$

则算法 (2) 中的 $\widetilde{u}_{j}^{n}$ 具有如下形式,

$$
\widetilde{u}_{j}^{n}=\Pi\left(u^{n} ; x_{j}^{n}, x_{j+1}^{n}\right)\left(x_{j}^{n+1}\right) .
$$


在时间段 $\left[t_{n-1}, t_{n}\right)$ 内及空间网格 $x^{n}$ 上, 定义如下网格依赖 $L_{2}$ 范数

$$
\|\psi\|_{n}=\left(\sum_{j}\left(\psi\left(x_{j}^{n}\right)\right)^{2} \frac{x_{j+1}^{n}-x_{j-1}^{n}}{2}\right)^{1 / 2},
$$

和网格依赖半范数

$$
\|\| \psi \|_{n}=\left(\sum_{j}\left(\frac{\psi\left(x_{j+1}^{n}\right)-\psi\left(x_{j}^{n}\right)}{x_{j+1}^{n}-x_{j}^{n}}\right)^{2}\left(x_{j+1}^{n}-x_{j}^{n}\right)\right)^{1 / 2} .
$$

在这些范数下, 我们给出如下收玫性定理:

定理 2 假定 $(\mathrm{C} 1)-(\mathrm{C} 3)$ 成立, 则带线性插值 $(22)$ 的移动网格重构有限差分算法 (2) 的误差估 计如下:

$$
\begin{aligned}
\left\|u^{m}-u\left(x, t_{m}\right)\right\|_{m} \leqslant & C \exp (C T)\left\{\left\|u^{0}-u_{0}(x)\right\|_{0}+\Delta t_{0} \frac{\left\|e^{0}\right\|_{0}^{2}}{\left\|e^{0}\right\|_{0}}\right. \\
& +\sum_{0 \leqslant n \leqslant m-1} \Delta t_{n}\left[\sum_{j}\left(\tau_{j}^{n+1}\right)^{2} \frac{x_{j+1}^{n+1}-x_{j-1}^{n+1}}{2}\right]^{1 / 2} \\
& \left.+\sum_{0 \leqslant n \leqslant m-1}\left\|\Pi\left(u\left(\cdot, t_{n}\right) ; x_{j}^{n}, x_{j+1}^{n}\right)(x)-u\left(x, t_{n}\right)\right\|_{n+1}\right\} .
\end{aligned}
$$

证明 令

$$
e_{j}^{n} \equiv u_{j}^{n}-u\left(x_{j}^{n}, t_{n}\right), \quad \widetilde{e}_{j}^{n} \equiv \widetilde{u}_{j}^{n}-u\left(x_{j}^{n+1}, t_{n}\right) .
$$

由 (17) 减 (2), 得误差方程

$$
\frac{e_{j}^{n+1}-\widetilde{e}_{j}^{n}}{\Delta t_{n}}=\frac{2}{x_{j+1}^{n+1}-x_{j-1}^{n+1}}\left[\frac{e_{j+1}^{n+1}-e_{j}^{n+1}}{x_{j+1}^{n+1}-x_{j}^{n+1}}-\frac{e_{j}^{n+1}-e_{j-1}^{n+1}}{x_{j}^{n+1}-x_{j-1}^{n+1}}\right]-\tau_{j}^{n+1} .
$$

将此方程两边同乘以 $e_{j}^{n+1}\left(x_{j+1}^{n+1}-x_{j-1}^{n+1}\right) / 2$, 然后对指标 $j$ 进行求和, 可得

$$
\begin{aligned}
\sum_{j} \frac{\left(e_{j}^{n+1}\right)^{2}-\widetilde{e}_{j}^{n} e_{j}^{n+1}}{\Delta t_{n}} \frac{x_{j+1}^{n+1}-x_{j-1}^{n+1}}{2}= & \sum_{j} e_{j}^{n+1}\left[\frac{e_{j+1}^{n+1}-e_{j}^{n+1}}{x_{j+1}^{n+1}-x_{j}^{n+1}}-\frac{e_{j}^{n+1}-e_{j-1}^{n+1}}{x_{j}^{n+1}-x_{j-1}^{n+1}}\right] \\
& -\sum_{j} \tau_{j}^{n+1} e_{j}^{n+1} \frac{x_{j+1}^{n+1}-x_{j-1}^{n+1}}{2}
\end{aligned}
$$

其等价形式为

$$
\begin{aligned}
\sum_{j}\left(e_{j}^{n+1}\right)^{2} \frac{x_{j+1}^{n+1}-x_{j-1}^{n+1}}{2}= & \sum_{j} \widetilde{e}_{j}^{n} e_{j}^{n+1} \frac{x_{j+1}^{n+1}-x_{j-1}^{n+1}}{2}-\Delta t_{n} \sum_{j} \frac{\left(e_{j+1}^{n+1}-e_{j}^{n+1}\right)^{2}}{x_{j+1}^{n+1}-x_{j}^{n+1}} \\
& -\Delta t_{n} \sum_{j} \tau_{j}^{n+1} e_{j}^{n+1} \frac{x_{j+1}^{n+1}-x_{j-1}^{n+1}}{2} .
\end{aligned}
$$

应用 Schwarz 不等式可得

$$
\left|\sum_{j} \widetilde{e}_{j}^{n} e_{j}^{n+1} \frac{x_{j+1}^{n+1}-x_{j-1}^{n+1}}{2}\right| \leqslant\left[\sum_{j}\left(e_{j}^{n+1}\right)^{2} \frac{x_{j+1}^{n+1}-x_{j-1}^{n+1}}{2}\right]^{\frac{1}{2}}\left[\sum_{j}\left(\widetilde{e}_{j}^{n}\right)^{2} \frac{x_{j+1}^{n+1}-x_{j-1}^{n+1}}{2}\right]^{\frac{1}{2}}
$$


和

$$
\left|\tau_{j}^{n+1} e_{j}^{n+1} \frac{x_{j+1}^{n+1}-x_{j-1}^{n+1}}{2}\right| \leqslant\left[\sum_{j}\left(e_{j}^{n+1}\right)^{2} \frac{x_{j+1}^{n+1}-x_{j-1}^{n+1}}{2}\right]^{\frac{1}{2}}\left[\sum_{j}\left(\tau_{j}^{n+1}\right)^{2} \frac{x_{j+1}^{n+1}-x_{j-1}^{n+1}}{2}\right]^{\frac{1}{2}} .
$$

再由 (25), 我们得到

$$
\begin{gathered}
{\left[\sum_{j}\left(e_{j}^{n+1}\right)^{2} \frac{x_{j+1}^{n+1}-x_{j-1}^{n+1}}{2}\right]^{\frac{1}{2}}+\frac{\Delta t_{n}}{\left[\sum_{j}\left(e_{j}^{n+1}\right)^{2} \frac{\left(x_{j+1}^{n+1}-x_{j-1}^{n+1}\right)}{2}\right]^{\frac{1}{2}}} \sum_{j} \frac{\left(e_{j+1}^{n+1}-e_{j}^{n+1}\right)^{2}}{x_{j+1}^{n+1}-x_{j}^{n+1}}} \\
\leqslant\left[\sum_{j}\left(\widetilde{e}_{j}^{n}\right)^{2} \frac{x_{j+1}^{n+1}-x_{j-1}^{n+1}}{2}\right]^{\frac{1}{2}}+\Delta t_{n}\left[\sum_{j}\left(\tau_{j}^{n+1}\right)^{2} \frac{x_{j+1}^{n+1}-x_{j-1}^{n+1}}{2}\right]^{\frac{1}{2}} .
\end{gathered}
$$

由于

$$
\begin{aligned}
\widetilde{e}_{j}^{n}= & \widetilde{u}_{j}^{n}-u\left(x_{j}^{n+1}, t_{n}\right) \\
= & {\left[\Pi\left(u^{n} ; x_{j}^{n}, x_{j+1}^{n}\right)\left(x_{j}^{n+1}\right)-\Pi\left(u\left(\cdot, t_{n}\right) ; x_{j}^{n}, x_{j+1}^{n}\right)\left(x_{j}^{n+1}\right)\right] } \\
& +\left[\Pi\left(u\left(\cdot, t_{n}\right) ; x_{j}^{n}, x_{j+1}^{n}\right)\left(x_{j}^{n+1}\right)-u\left(x_{j}^{n+1}, t_{n}\right)\right]
\end{aligned}
$$

和

$$
\begin{aligned}
\Pi\left(u^{n} ;\right. & \left.x_{j}^{n}, x_{j+1}^{n}\right)\left(x_{j}^{n+1}\right)-\Pi\left(u\left(\cdot, t_{n}\right) ; x_{j}^{n}, x_{j+1}^{n}\right)\left(x_{j}^{n+1}\right) \\
& =\Pi\left(e^{n} ; x_{j}^{n}, x_{j+1}^{n}\right)\left(x_{j}^{n+1}\right) \\
& =\frac{x_{j+1}^{n}-x_{j}^{n+1}}{x_{j+1}^{n}-x_{j}^{n}} e_{j}^{n}+\frac{x_{j}^{n+1}-x_{j}^{n}}{x_{j+1}^{n}-x_{j}^{n}} e_{j+1}^{n} \\
& =e_{j}^{n}+\left(\frac{e_{j+1}^{n}-e_{j}^{n}}{x_{j+1}^{n}-x_{j}^{n}}\right)\left(x_{j}^{n+1}-x_{j}^{n}\right),
\end{aligned}
$$

使用下面的 Minkowski 不等式:

$$
\left(\sum_{j} \omega_{j}\left|a_{j}+b_{j}\right|^{p}\right)^{1 / p} \leqslant\left(\sum_{j} \omega_{j}\left|a_{j}\right|^{p}\right)^{1 / p}+\left(\sum_{j} \omega_{j}\left|b_{j}\right|^{p}\right)^{1 / p}, \quad 1 \leqslant p<\infty,
$$

我们得到

$$
\begin{aligned}
& \left(\sum_{j}\left(\widetilde{e}_{j}^{n}\right)^{2} \frac{x_{j+1}^{n+1}-x_{j-1}^{n+1}}{2}\right)^{1 / 2} \\
& \leqslant\left(\sum_{j}\left(e_{j}^{n}\right)^{2} \frac{x_{j+1}^{n+1}-x_{j-1}^{n+1}}{2}\right)^{1 / 2}+\left(\sum_{j}\left(x_{j}^{n+1}-x_{j}^{n}\right)^{2}\left(\frac{e_{j+1}^{n}-e_{j}^{n}}{x_{j+1}^{n}-x_{j}^{n}}\right)^{2} \frac{x_{j+1}^{n+1}-x_{j-1}^{n+1}}{2}\right)^{1 / 2} \\
& \quad+\left[\sum_{j}\left[\Pi\left(u\left(\cdot, t_{n}\right) ; x_{j}^{n}, x_{j+1}^{n}\right)\left(x_{j}^{n+1}\right)-u\left(x_{j}^{n+1}, t_{n}\right)\right]^{2} \frac{x_{j+1}^{n+1}-x_{j-1}^{n+1}}{2}\right]^{1 / 2} \\
& =:(\mathrm{I})+(\mathrm{II})+(\mathrm{III}) .
\end{aligned}
$$

应用条件 (C1) 得到下面估计:

$$
(\mathrm{I}) \leqslant \sqrt{1+C \Delta t_{n}}\left(\sum_{j}\left(e_{j}^{n}\right)^{2} \frac{x_{j+1}^{n}-x_{j-1}^{n}}{2}\right)^{1 / 2} \leqslant\left(1+C \Delta t_{n}\right)\left\|e^{n}\right\|_{n},
$$




$$
\begin{aligned}
(\mathrm{II}) & \leqslant C \Delta t_{n}\left(1+C \Delta t_{n}\right)\left(\sum_{j}\left(\frac{e_{j+1}^{n}-e_{j}^{n}}{x_{j+1}^{n}-x_{j}^{n}}\right)^{2} \frac{x_{j+1}^{n}-x_{j-1}^{n}}{2}\right)^{1 / 2} \\
& \leqslant C \Delta t_{n}\left(1+C \Delta t_{n}\right)\left(\sum_{j}\left(\frac{e_{j+1}^{n}-e_{j}^{n}}{x_{j+1}^{n}-x_{j}^{n}}\right)^{2}\left(x_{j+1}^{n}-x_{j}^{n}\right)\right)^{1 / 2} \\
& =C \Delta t_{n}\left(1+C \Delta t_{n}\right) \frac{\left\|e^{n}\right\| \|_{n}}{\left.\left\|e^{n}\right\|\right|_{n} ^{1 / 2}}\left\|^{n}\right\|_{n}^{1 / 2} \\
& \leqslant C \Delta t_{n}\left(1+C \Delta t_{n}\right)\left[\frac{\sigma}{2} \frac{\left\|e^{n}\right\|_{n}^{2}}{\left\|e^{n}\right\|_{n}}+\frac{1}{\sigma} \Delta t_{n}\left\|e^{n}\right\|_{n}\right] \\
& \leqslant C \sigma \Delta t_{n}\left(1+C \Delta t_{n}\right) \frac{\left\|e^{n}\right\| \|_{n}^{2}}{\left\|e^{n}\right\|_{n}}+\frac{C}{\sigma} \Delta t_{n}\left\|e^{n}\right\|_{n},
\end{aligned}
$$

其中第一个不等式由条件 $(\mathrm{C} 1)$ 和 $(\mathrm{C} 2)$ 得到, 第二个不等式由 (19) 得到, $\sigma$ 是一个任意正数. 将 $(30)$, (31) 和

$$
(\mathrm{III})=\left\|\Pi\left(u\left(\cdot, t_{n}\right) ; x_{j}^{n}, x_{j+1}^{n}\right)(x)-u\left(x, t_{n}\right)\right\|_{n+1}
$$

代入 (29), 我们得到

$$
\begin{aligned}
\left(\sum_{j}\left(\widetilde{e}_{j}^{n}\right)^{2} \frac{\left(x_{j+1}^{n+1}-x_{j-1}^{n+1}\right)}{2}\right)^{1 / 2} \leqslant & \left(1+C\left(1+\frac{1}{\sigma}\right) \Delta t_{n}\right)\left\|e^{n}\right\|_{n}+C \sigma \Delta t_{n}\left(1+C \Delta t_{n}\right) \frac{\left\|e^{n}\right\| \|_{n}^{2}}{\left\|e^{n}\right\|_{n}} \\
& +C\left\|\Pi\left(u\left(\cdot, t_{n}\right) ; x_{j}^{n}, x_{j+1}^{n}\right)(x)-u\left(x, t_{n}\right)\right\|_{n+1} .
\end{aligned}
$$

注意正常数 $C$ 与 $\sigma$ 相互无关, 我们可以取 $\sigma$ 使得 $1 /(C \sigma) \geqslant 1$ (例如 $1 /(C \sigma)=2$ ). 于是只要取较小的 时间步长满足条件

我们就可通过 (26) 和 (32) 得出

$$
\Delta t_{n+1} \leqslant \frac{1}{C \sigma} \Delta t_{n}
$$

$$
\begin{aligned}
\left\|e^{n+1}\right\|_{n+1}+C \sigma \Delta t_{n+1} \frac{\left\|e^{n+1}\right\| \|_{n+1}^{2}}{\left\|e^{n+1}\right\|_{n+1}} \leqslant & \left(1+C\left(1+\frac{1}{\sigma}\right) \Delta t_{n}\right)\left(\left\|e^{n}\right\|_{n}+C \sigma \Delta t_{n} \frac{\left\|e^{n}\right\| \|_{n}^{2}}{\left\|e^{n}\right\|_{n}}\right) \\
& +\Delta t_{n}\left[\sum_{j}\left(\tau_{j}^{n+1}\right)^{2} \frac{x_{j+1}^{n+1}-x_{j-1}^{n+1}}{2}\right]^{\frac{1}{2}} \\
& +C\left\|\Pi\left(u\left(\cdot, t_{n}\right) ; x_{j}^{n}, x_{j+1}^{n}\right)(x)-u\left(x, t_{n}\right)\right\|_{n+1} .
\end{aligned}
$$

最后由离散的 Gronwall 不等式 (引理 2 ) 及 $C \sigma \leqslant 1$, 我们就得到误差估计 (23).

定理 3 在定理 2 的条件下, 带线性插值 (22) 的移动网格重构有限差分算法 (2) 的收玫阶为

$$
\max _{m}\left\|u^{m}-u\left(x, t_{m}\right)\right\|_{m}=\max \left\{O\left(N^{-1}\right), O(\tau)\right\},
$$

这里 $N$ 是空间网格点数, 并且 $\tau=\max \left\{\Delta t_{n}\right\}$.

证明 线性插值的误差为

$$
\Pi\left(u\left(\cdot, t_{n}\right) ; x_{j}^{n}, x_{j+1}^{n}\right)(x)-u\left(x, t_{n}\right)=\left(x-x_{j}^{n}\right)\left(x-x_{j+1}^{n}\right) u\left[x_{j}^{n}, x_{j+1}^{n}, x\right],
$$

这里 $u[\cdot, \ldots, \cdot]$ 表示插商. 因此我们有如下估计:

$$
\left\|\Pi\left(u\left(\cdot, t_{n}\right) ; x_{j}^{n}, x_{j+1}^{n}\right)(x)-u\left(x, t_{n}\right)\right\|_{n+1}
$$




$$
\begin{aligned}
& =\left[\sum_{j}\left[\Pi\left(u\left(\cdot, t_{n}\right) ; x_{j}^{n}, x_{j+1}^{n}\right)\left(x_{j}^{n+1}\right)-u\left(x_{j}^{n+1}, t_{n}\right)\right]^{2} \frac{x_{j+1}^{n+1}-x_{j-1}^{n+1}}{2}\right]^{1 / 2} \\
& \leqslant C\left[\sum_{j}\left[\left(x_{j}^{n+1}-x_{j}^{n}\right)\left(x_{j}^{n+1}-x_{j+1}^{n}\right) u\left[x_{j}^{n}, x_{j+1}^{n}, x_{j}^{n+1}\right]\right]^{2} \frac{x_{j+1}^{n+1}-x_{j-1}^{n+1}}{2}\right]^{1 / 2} \\
& \leqslant C \Delta t_{n} N^{-1} .
\end{aligned}
$$

并且截断误差估计如下:

$$
\left[\sum_{j}\left(\tau_{j}^{n+1}\right)^{2} \frac{x_{j+1}^{n+1}-x_{j-1}^{n+1}}{2}\right]^{\frac{1}{2}}=\max \left\{O\left(N^{-2}\right), O(\tau)\right\} .
$$

另外,

$$
\begin{aligned}
\Delta t_{0}\left\|e^{0}\right\| \|_{0}^{2} & =\Delta t_{0} \sum_{j}\left(\frac{e_{j}^{0}-e_{j-1}^{0}}{x_{j+1}^{0}-x_{j}^{0}}\right)^{2}\left(x_{j+1}^{0}-x_{j}^{0}\right) \\
& \leqslant C \sum_{j}\left(e_{j}^{0}-e_{j-1}^{0}\right)^{2}\left(x_{j+1}^{0}-x_{j}^{0}\right) \\
& \leqslant C \sum_{j}\left(e_{j}^{0}\right)^{2} \frac{x_{j+1}^{0}-x_{j-1}^{0}}{2}=C\left\|e^{0}\right\|_{0}^{2},
\end{aligned}
$$

其中第一个不等式由 (C4) 得到. 因此使用 (23) 中各项的估计, 并注意到初始误差能够做到足够小 使得

$$
\left\|u^{0}-u_{0}(x)\right\|_{0}+\Delta t_{0} \frac{\left.\left\|e^{0}\right\|\right|_{0} ^{2}}{\left\|e^{0}\right\|_{0}} \leqslant C\left\|e^{0}\right\|_{0}
$$

成立, 我们就获得了定理中的结果.

\section{4 二次插值情形}

定义二次插值

$$
\begin{aligned}
\Pi\left(u^{n} ; x_{j-1}^{n}, x_{j}^{n}, x_{j+1}^{n}\right)(x)= & \frac{\left(x-x_{j}^{n}\right)\left(x-x_{j+1}^{n}\right)}{\left(x_{j-1}^{n}-x_{j}^{n}\right)\left(x_{j-1}^{n}-x_{j+1}^{n}\right)} u_{j-1}^{n}+\frac{\left(x-x_{j-1}^{n}\right)\left(x-x_{j+1}^{n}\right)}{\left(x_{j}^{n}-x_{j-1}^{n}\right)\left(x_{j}^{n}-x_{j+1}^{n}\right)} u_{j}^{n} \\
& +\frac{\left(x-x_{j}^{n}\right)\left(x-x_{j-1}^{n}\right)}{\left(x_{j+1}^{n}-x_{j}^{n}\right)\left(x_{j+1}^{n}-x_{j-1}^{n}\right)} u_{j+1}^{n} .
\end{aligned}
$$

于是 (2) 中的 $\widetilde{u}_{j}^{n}$ 可写为

$$
\widetilde{u}_{j}^{n}=\Pi\left(u^{n} ; x_{j-1}^{n}, x_{j}^{n}, x_{j+1}^{n}\right)\left(x_{j}^{n+1}\right) .
$$

定理 4 假定条件 (C1)-(C4) 成立, 则带二次插值 (41) 的移动网格重构有限差分算法 (2) 有如 下误差估计：

$$
\begin{aligned}
\left\|u^{m}-u\left(x, t_{m}\right)\right\|_{m} \leqslant & C \exp (C T)\left\{\left\|u^{0}-u_{0}(x)\right\|_{0}+\Delta t_{0} \frac{\left\|e^{0}\right\|_{0}^{2}}{\left\|e^{0}\right\|_{0}}\right. \\
& +\sum_{0 \leqslant n \leqslant m-1} \Delta t_{n}\left[\sum_{j}\left(\tau_{j}^{n+1}\right)^{2} \frac{x_{j+1}^{n+1}-x_{j-1}^{n+1}}{2}\right]^{1 / 2}
\end{aligned}
$$




$$
\left.+\sum_{0 \leqslant n \leqslant m-1}\left\|\Pi\left(u\left(\cdot, t_{n}\right) ; x_{j-1}^{n}, x_{j}^{n}, x_{j+1}^{n}\right)(x)-u\left(x, t_{n}\right)\right\|_{n+1}\right\} .
$$

而且算法具有收敛阶

$$
\max _{m}\left\|u^{m}-u\left(x, t_{m}\right)\right\|_{m}=\max \left\{O\left(N^{-2}\right), O(\tau)\right\} .
$$

证明 与定理 2 的证明过程类似, 我们可得关系式 (26), 其中 $\widetilde{e}_{j}^{n}$ 为

$$
\begin{aligned}
\widetilde{e}_{j}^{n}= & \widetilde{u}_{j}^{n}-u\left(x_{j}^{n+1}, t_{n}\right) \\
= & {\left[\Pi\left(u^{n} ; x_{j-1}^{n}, x_{j}^{n}, x_{j+1}^{n}\right)\left(x_{j}^{n+1}\right)-\Pi\left(u\left(\cdot, t_{n}\right) ; x_{j-1}^{n}, x_{j}^{n}, x_{j+1}^{n}\right)\left(x_{j}^{n+1}\right)\right] } \\
& +\left[\Pi\left(u\left(\cdot, t_{n}\right) ; x_{j-1}^{n}, x_{j}^{n}, x_{j+1}^{n}\right)\left(x_{j}^{n+1}\right)-u\left(x_{j}^{n+1}, t_{n}\right)\right] .
\end{aligned}
$$

由于

$$
\begin{gathered}
\Pi\left(u^{n} ; x_{j-1}^{n}, x_{j}^{n}, x_{j+1}^{n}\right)\left(x_{j}^{n+1}\right)-\Pi\left(u\left(\cdot, t_{n}\right) ; x_{j-1}^{n}, x_{j}^{n}, x_{j+1}^{n}\right)\left(x_{j}^{n+1}\right) \\
=e_{j}^{n}+\frac{\left(x_{j}^{n+1}-x_{j}^{n}\right)\left(x_{j}^{n+1}-x_{j+1}^{n}\right)}{x_{j-1}^{n}-x_{j+1}^{n}} \frac{e_{j}^{n}-e_{j-1}^{n}}{x_{j}^{n}-x_{j-1}^{n}} \\
\quad+\frac{\left(x_{j}^{n+1}-x_{j}^{n}\right)\left(x_{j}^{n+1}-x_{j-1}^{n}\right)}{x_{j+1}^{n}-x_{j-1}^{n}} \frac{e_{j+1}^{n}-e_{j}^{n}}{x_{j+1}^{n}-x_{j}^{n}} .
\end{gathered}
$$

与定理 2 中 (29) 的推导类似, 我们可推得

$$
\begin{aligned}
\left(\sum_{j}\left(\widetilde{e}_{j}^{n}\right)^{2}\right. & \left.\frac{x_{j+1}^{n+1}-x_{j-1}^{n+1}}{2}\right)^{1 / 2} \\
\leqslant & \left(1+C \Delta t_{n}\right)\left\|e^{n}\right\|_{n}+\left\|\Pi\left(u\left(\cdot, t_{n}\right) ; x_{j-1}^{n}, x_{j}^{n}, x_{j+1}^{n}\right)(x)-u\left(x, t_{n}\right)\right\|_{n+1} \\
+ & {\left[\sum_{j}\left(x_{j}^{n+1}-x_{j}^{n}\right)^{2}\left(\frac{x_{j}^{n+1}-x_{j+1}^{n}}{x_{j-1}^{n}-x_{j+1}^{n}}\right)^{2}\left(\frac{e_{j}^{n}-e_{j-1}^{n}}{x_{j}^{n}-x_{j-1}^{n}}\right)^{2} \frac{x_{j+1}^{n+1}-x_{j-1}^{n+1}}{2}\right]^{1 / 2} } \\
+ & {\left[\sum_{j}\left(x_{j}^{n+1}-x_{j}^{n}\right)^{2}\left(\frac{x_{j}^{n+1}-x_{j-1}^{n}}{x_{j+1}^{n}-x_{j-1}^{n}}\right)^{2}\left(\frac{e_{j+1}^{n}-e_{j}^{n}}{x_{j+1}^{n}-x_{j}^{n}}\right)^{2} \frac{x_{j+1}^{n+1}-x_{j-1}^{n+1}}{2}\right]^{1 / 2} . }
\end{aligned}
$$

接下来我们估计 (46) 中的最后两项. 由条件 $(\mathrm{C} 1),(\mathrm{C} 2),(\mathrm{C} 4)$, 及引理 1 中的不等式 (18) 和 (19), 我们 得到

$$
\begin{aligned}
& {\left[\sum_{j}\left(x_{j}^{n+1}-x_{j}^{n}\right)^{2}\left(\frac{x_{j}^{n+1}-x_{j+1}^{n}}{x_{j-1}^{n}-x_{j+1}^{n}}\right)^{2}\left(\frac{e_{j}^{n}-e_{j-1}^{n}}{x_{j}^{n}-x_{j-1}^{n}}\right)^{2} \frac{x_{j+1}^{n+1}-x_{j-1}^{n+1}}{2}\right]^{1 / 2}} \\
& \quad \leqslant\left[\sum_{j}\left(x_{j}^{n+1}-x_{j}^{n}\right)^{2}\left(1+\frac{\left|x_{j}^{n+1}-x_{j}^{n}\right|}{x_{j+1}^{n}-x_{j}^{n}}\right)^{2}\left(\frac{e_{j}^{n}-e_{j-1}^{n}}{x_{j}^{n}-x_{j-1}^{n}}\right)^{2} \frac{x_{j+1}^{n+1}-x_{j-1}^{n+1}}{2}\right]^{1 / 2} \\
& \quad \leqslant C \Delta t_{n}\left(1+C \Delta t_{n}\right)\left[\sum_{j}\left(\frac{e_{j}^{n}-e_{j-1}^{n}}{x_{j}^{n}-x_{j-1}^{n}}\right)^{2}\left(x_{j}^{n}-x_{j-1}^{n}\right)\right]^{1 / 2} \\
& \quad=C \Delta t_{n}\left(1+C \Delta t_{n}\right)\left(\frac{\left\|e^{n}\right\| \|_{n}}{\left\|e^{n}\right\|_{n}^{1 / 2}}\right)\left(\left\|e^{n}\right\|_{n}^{1 / 2}\right) \\
& \leqslant C \Delta t_{n}\left(1+C \Delta t_{n}\right)\left[\frac{\sigma}{2} \frac{\left\|e^{n}\right\|_{n}^{2}}{\left\|e^{n}\right\|_{n}}+\frac{1}{\sigma}\left\|e^{n}\right\|_{n}\right]
\end{aligned}
$$




$$
\leqslant \frac{\sigma}{2} C \Delta t_{n}\left(1+C \Delta t_{n}\right) \frac{\left\|e^{n}\right\|_{n}^{2}}{\left\|e^{n}\right\|_{n}}+\frac{C \Delta t_{n}}{\sigma}\left\|e^{n}\right\|_{n}
$$

类似地, 我们得到

$$
\begin{aligned}
& {\left[\sum_{j}\left(x_{j}^{n+1}-x_{j}^{n}\right)^{2}\left(\frac{x_{j}^{n+1}-x_{j-1}^{n}}{x_{j+1}^{n}-x_{j-1}^{n}}\right)^{2}\left(\frac{e_{j+1}^{n}-e_{j}^{n}}{x_{j+1}^{n}-x_{j}^{n}}\right)^{2} \frac{x_{j+1}^{n+1}-x_{j-1}^{n+1}}{2}\right]^{1 / 2}} \\
& \leqslant \frac{\sigma}{2} C \Delta t_{n}\left(1+C \Delta t_{n}\right) \frac{\left.\left\|e^{n}\right\|\right|_{n} ^{2}}{\left\|e^{n}\right\|_{n}}+\frac{C \Delta t_{n}}{\sigma}\left\|e^{n}\right\|_{n}
\end{aligned}
$$

因此有

$$
\begin{aligned}
\left(\sum_{j}\left(\widetilde{e}_{j}^{n}\right)^{2} \frac{x_{j+1}^{n+1}-x_{j-1}^{n+1}}{2}\right)^{1 / 2} \leqslant & \left(1+C\left(1+\frac{2}{\sigma}\right) \Delta t_{n}\right)\left(\left\|e^{n}\right\|_{n}+C \sigma \Delta t_{n} \frac{\left\|e^{n}\right\| \|_{n}^{2}}{\left\|e^{n}\right\|_{n}}\right) \\
& +\left\|\Pi\left(u\left(\cdot, t_{n}\right) ; x_{j-1}^{n}, x_{j}^{n}, x_{j+1}^{n}\right)(x)-u\left(x, t_{n}\right)\right\|_{n+1} .
\end{aligned}
$$

把 (49) 用到 (26) 中, 并且假定

我们得到

$$
\Delta t_{n+1} \leqslant \frac{1}{C \sigma} \Delta t_{n}
$$

$$
\begin{aligned}
\left\|e^{n+1}\right\|_{n+1}+C \sigma \Delta t_{n+1} \frac{\left\|e^{n+1}\right\| \|_{n+1}^{2}}{\left\|e^{n+1}\right\|_{n+1}} \leqslant & \left(1+C\left(1+\frac{2}{\sigma}\right) \Delta t_{n}\right)\left(\left\|e^{n}\right\|_{n}+C \sigma \Delta t_{n} \frac{\left\|e^{n}\right\| \|_{n}^{2}}{\left\|e^{n}\right\|_{n}}\right) \\
& +\Delta t_{n}\left[\sum_{j}\left(\tau_{j}^{n+1}\right)^{2} \frac{x_{j+1}^{n+1}-x_{j-1}^{n+1}}{2}\right]^{\frac{1}{2}} \\
& +C\left\|\Pi\left(u\left(\cdot, t_{n}\right) ; x_{j-1}^{n}, x_{j}^{n}, x_{j+1}^{n}\right)(x)-u\left(x, t_{n}\right)\right\|_{n+1} .
\end{aligned}
$$

于是由离散的 Gronwall 不等式 (引理 2) 可得 (42). 最后收敛阶 (43) 可由 (37), (39) 及下面估计得到,

$$
\begin{aligned}
\| & \Pi\left(u\left(\cdot, t_{n}\right) ; x_{j-1}^{n}, x_{j}^{n}, x_{j+1}^{n}\right)(x)-u\left(x, t_{n}\right) \|_{n+1} \\
& =\left[\sum_{j}\left[\Pi\left(u\left(\cdot, t_{n}\right) ; x_{j-1}^{n}, x_{j}^{n}, x_{j+1}^{n}\right)\left(x_{j}^{n+1}\right)-u\left(x_{j}^{n+1}, t_{n}\right)\right]^{2} \frac{x_{j+1}^{n+1}-x_{j-1}^{n+1}}{2}\right]^{1 / 2} \\
& \leqslant C\left[\sum_{j}\left[\left(x_{j}^{n+1}-x_{j-1}^{n}\right)\left(x_{j}^{n+1}-x_{j}^{n}\right)\left(x_{j}^{n+1}-x_{j+1}^{n}\right) u\left[x_{j-1}^{n}, x_{j}^{n}, x_{j+1}^{n}, x_{j}^{n+1}\right]\right]^{2} \frac{x_{j+1}^{n+1}-x_{j-1}^{n+1}}{2}\right]^{1 / 2} \\
& =\Delta t_{n} \max \left\{O\left(N^{-2}\right), O(\tau)\right\} .
\end{aligned}
$$

\section{5 数值算例}

例 1 考虑线性方程

$$
u_{t}-u_{x x}=\delta\left(x-x_{0}(t)\right)(2+t+x), \quad x \in[-5,5], \quad t \in[0,1],
$$

并且给定 $x_{0}(t)=2 t$ 及初始条件

$$
u(x, 0)= \begin{cases}\cos ^{2}(\pi x / 2), & -1<x<1 ; \\ 0, & -5 \leqslant x \leqslant-1 \text { 或 } 1 \leqslant x \leqslant 5 .\end{cases}
$$


在实验中, 时间上我们选择变级网格 (graded mesh) $t_{n}=(n / L)^{2}, n=0,1, \ldots, L$. 在时刻 $t_{n}$, 定义坐标 变换

$$
\xi=\Gamma(x)= \begin{cases}-\sqrt{x_{0}\left(t_{n}\right)-x}, & -5 \leqslant x<x_{0}\left(t_{n}\right) \\ \sqrt{x-x_{0}\left(t_{n}\right)}, & x_{0}\left(t_{n}\right) \leqslant x<5\end{cases}
$$

令

$$
\xi_{j}=\Gamma(-5)+j \times \frac{\Gamma(5)-\Gamma(-5)}{N}, \quad j=0,1, \ldots, N .
$$

则在 $t_{n}$ 时刻, 自适应变级网格给出如下:

$$
x_{j}^{n}=\Gamma^{-1}\left(\xi_{j}\right), \quad j=0,1, \ldots, N .
$$

如此选择空间网格的动机是上面的自适应变级网格具有下面性质:

(i) 若 $x_{0}\left(t_{n}\right) \in\left[x_{j-1}^{n}, x_{j+1}^{n}\right]$, 则有 $\max \left\{h_{j}^{n}, h_{j+1}^{n}\right\}=O\left(1 / N^{2}\right)$;

(ii) $h_{j}^{n}-h_{j-1}^{n}=O\left(1 / N^{2}\right)$.

由定理 1 , 我们知道

$$
\left|\tau_{j}^{n+1}\right| \leqslant \begin{cases}C \max \left\{h_{j+1}^{n+1}-h_{j}^{n+1},\left(h_{j+1}^{n+1}\right)^{2}+\left(h_{j}^{n+1}\right)^{2}, \Delta t\right\}, & \text { 若 } x_{0}\left(t_{n+1}\right) \notin\left[x_{j-1}^{n+1}, x_{j+1}^{n+1}\right] ; \\ C \max \left\{h_{j+1}^{n+1}+h_{j}^{n+1}, \Delta t\right\}, & \text { 若 } x_{0}\left(t_{n+1}\right) \in\left[x_{j-1}^{n+1}, x_{j+1}^{n+1}\right] .\end{cases}
$$

根据定理 3 和 4 中的结果, 使用线性插值时收玫阶为一阶, 使用二次插值时收敛阶为二阶, 即

$$
\max _{n}\left\|u\left(x, t_{n}\right)-u_{N, L}^{n}\left(x, t_{n}\right)\right\|_{n} \leqslant C N^{-r},
$$

其中 $r=1$ 或者 $r=2$. 于是有,

$$
\begin{aligned}
\max _{n}\left\|e_{N, L}^{n}\right\|_{n} & \equiv \max _{n}\left\|u_{N, L}^{n}\left(x, t_{n}\right)-u_{2 N, 2 L}^{n}\left(x, t_{n}\right)\right\|_{n} \\
& \leqslant \max _{n}\left\|u\left(x, t_{n}\right)-u_{N, L}^{n}\left(x, t_{n}\right)\right\|_{n}+\max _{n}\left\|u\left(x, t_{n}\right)-u_{2 N, 2 L}^{n}\left(x, t_{n}\right)\right\|_{n} \leqslant C N^{-r},
\end{aligned}
$$

并且

$$
\frac{\max _{n}\left\|e_{2 N, 2 L}^{n}\right\|_{n}}{\max _{n}\left\|e_{N, L}^{n}\right\|_{n}} \approx \frac{(2 N)^{-r}}{N^{-r}}=2^{-r}
$$

于是 $r$ 可定义为

$$
r=\left|\log _{2}\left(\frac{\max _{n}\left\|e_{2 N, 2 L}^{n}\right\|_{n}}{\max _{n}\left\|e_{N, L}^{n}\right\|_{n}}\right)\right| .
$$

我们还将计算无穷范数误差

$$
\max _{n}\left\|e_{N, L}^{n}\right\|_{\infty} \equiv \max _{n}\left\|u_{N, L}^{n}\left(x, t_{n}\right)-u_{2 N, 2 L}^{n}\left(x, t_{n}\right)\right\|_{\infty} .
$$

表 1 中数据验证了当使用线性插值时, 自适应网格重构算法的收敛阶为一阶 (即 $r=1$ ), 使用二次插 值时算法的收玫阶为二阶 (即 $r=2$ ), 实验结果与理论分析结果完全一致。

例 2 考虑非线性方程

$$
u_{t}-u_{x x}=\delta\left(x-x_{0}(t)\right)\left(1+u^{2}\right), \quad x \in[-5,5],
$$


表 1 例 1 的误差及收敛阶

\begin{tabular}{|c|c|c|c|c|c|c|}
\hline 线性插值 & & & & & & \\
\hline$N, L$ & 20,25 & 40,100 & 80,400 & 160,1600 & 320,6400 & 640,25600 \\
\hline $\max _{n}\left\|e_{N, L}^{n}\right\|_{n}$ & $1.0147 \mathrm{e}-1$ & $5.9625 \mathrm{e}-2$ & $3.8777 \mathrm{e}-2$ & $2.2220 \mathrm{e}-2$ & $1.1835 \mathrm{e}-2$ & \\
\hline$r$ & 0.8 & 0.6 & 0.8 & 0.9 & & \\
\hline $\max _{n}\left\|e_{N, L}^{n}\right\|_{\infty}$ & $9.2676 \mathrm{e}-2$ & $4.9878 \mathrm{e}-2$ & $3.0001 \mathrm{e}-2$ & $1.7424 \mathrm{e}-2$ & $9.5706 \mathrm{e}-3$ & \\
\hline$r$ & 0.9 & 0.7 & 0.8 & 0.9 & & \\
\hline \multicolumn{7}{|l|}{ 二次插值 } \\
\hline $\max _{n}\left\|e_{N, L}^{n}\right\|_{n}$ & $7.0062 \mathrm{e}-2$ & $3.3176 \mathrm{e}-2$ & $9.7300 \mathrm{e}-3$ & $2.5315 \mathrm{e}-3$ & $6.4003 \mathrm{e}-4$ & \\
\hline$r$ & 1.1 & 1.8 & 1.9 & 2.0 & & \\
\hline $\max _{n}\left\|e_{N, L}^{n}\right\|_{\infty}$ & $9.2676 \mathrm{e}-2$ & $4.9878 \mathrm{e}-2$ & $3.0001 \mathrm{e}-2$ & $1.7424 \mathrm{e}-2$ & $9.5706 \mathrm{e}-3$ & \\
\hline$r$ & 0.9 & 1.7 & 1.9 & 2.0 & & \\
\hline
\end{tabular}

表 2 例 2 的误差及收敛阶

\begin{tabular}{|c|c|c|c|c|c|c|}
\hline \multicolumn{7}{|l|}{ 线性插值 } \\
\hline$N, L$ & 20,40 & 40,160 & 80,640 & 160,2560 & 320,10240 & 640,40960 \\
\hline $\max _{n}\left\|e_{N, L}^{n}\right\|_{n}$ & $3.9813 \mathrm{e}-1$ & $1.1836 \mathrm{e}-1$ & $6.2152 \mathrm{e}-2$ & $3.2065 \mathrm{e}-2$ & $1.6064 \mathrm{e}-2$ & \\
\hline$r$ & 1.7 & 0.9 & 0.9 & 0.9 & & \\
\hline $\max _{n}\left\|e_{N, L}^{n}\right\|_{\infty}$ & $3.4408 \mathrm{e}-1$ & $9.6168 \mathrm{e}-2$ & $4.9507 \mathrm{e}-2$ & $2.5236 \mathrm{e}-2$ & $1.2612 \mathrm{e}-002$ & \\
\hline$r$ & 1.8 & 1.0 & 0.9 & 1.0 & & \\
\hline \multicolumn{7}{|l|}{ 二次插值 } \\
\hline $\max _{n}\left\|e_{N, L}^{n}\right\|_{n}$ & $2.7870 \mathrm{e}-1$ & $3.3263 \mathrm{e}-2$ & $5.4044 \mathrm{e}-3$ & $1.1923 \mathrm{e}-3$ & $2.9080 \mathrm{e}-4$ & \\
\hline$r$ & 3.0 & 2.6 & 2.2 & 2.0 & & \\
\hline $\max _{n}\left\|e_{N, L}^{n}\right\|_{\infty}$ & $2.1518 \mathrm{e}-1$ & $2.9150 \mathrm{e}-2$ & $5.2641 \mathrm{e}-3$ & $1.1871 \mathrm{e}-3$ & $2.7529 \mathrm{e}-4$ & \\
\hline$r$ & 2.8 & 2.4 & 2.1 & 2.1 & & \\
\hline
\end{tabular}

这里 $x_{0}=4 t, u(x, 0)=\cos ^{2}(\pi x / 10)$.

实验中, 时间上我们采用网格 $t_{n}=(n / L)^{2}, n=0,1, \ldots, L$, 空间网格通过求解移动网格偏微分方 程 MMPDE6 (见 $[22,18]$ ) 得到, 其中参数 $\tau=10^{-3}$, 监视函数取为

$$
M=\alpha\left|\frac{\partial u}{\partial x}\right|+(1-\alpha)\left(\left(x-x_{0}(t)\right)^{2}+\epsilon\right)^{-1 / 4},
$$

其中参数 $\alpha=0.1, \epsilon=10^{3} / N^{4}$. 表 2 中数据再次验证了本文的理论结果.

在最后一个例子中, 我们使用移动网格重构算法模拟爆破解.

例 3 考虑非线性爆破问题

$$
u_{t}-u_{x x}=\delta\left(x-x_{0}(t)\right)\left(1+u^{2}\right), \quad x \in[-5,5],
$$

其中 $x_{0}=k t, u(x, 0)=\cos ^{2}(\pi x / 10)$.

在数值求解过程中, 时间步长 $\Delta t_{n}=t_{n+1}-t_{n}$ 选取为

$$
\Delta t_{n}=\min \left(\frac{\mu}{\left(\max _{(j)}\left\{u_{j}^{n}\right\}\right)^{2}}, \mu\right),
$$



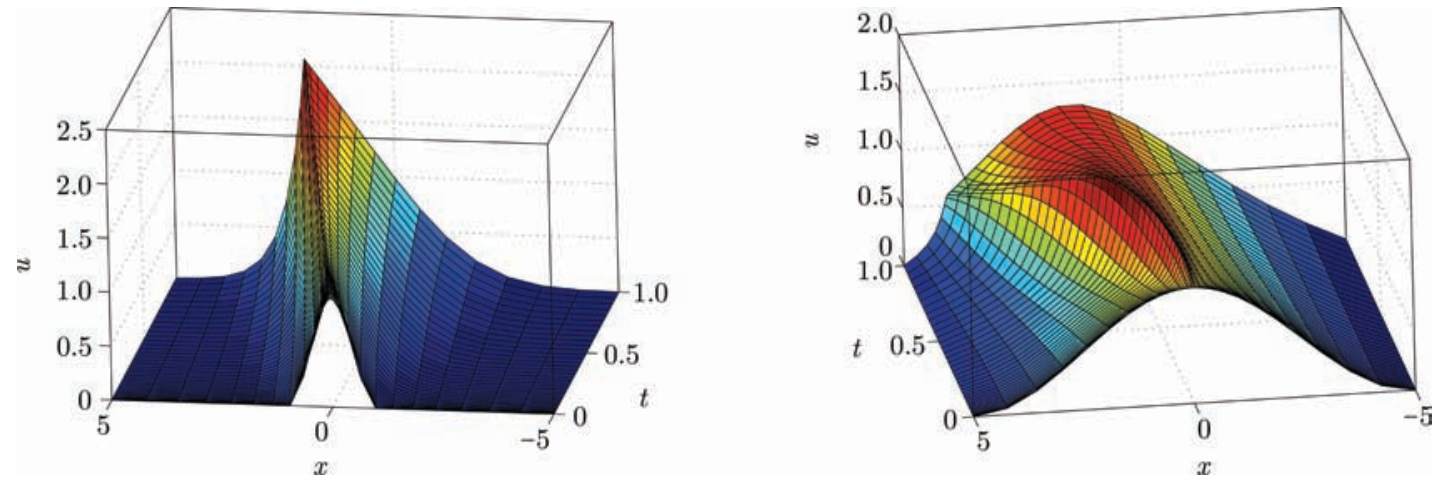

图 3 左图为例 1 的精确解, 右图为例 2 的精确解
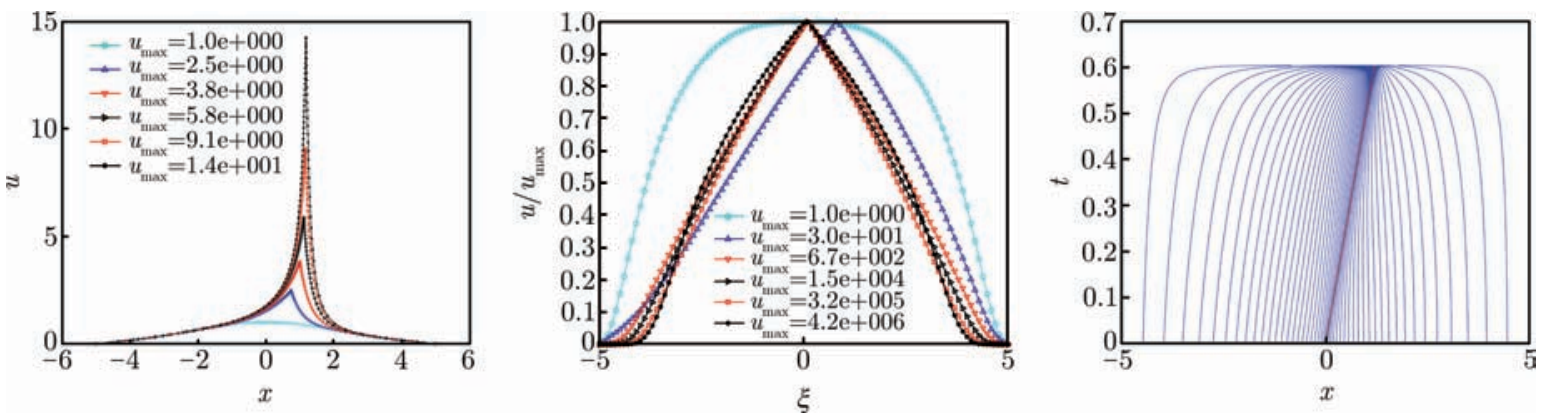

图 4 对于例 3 中 $k=2$ 情形, 从左到右分别为数值爆破解对物理变量和计算变量的图形以及移动网格轨迹图
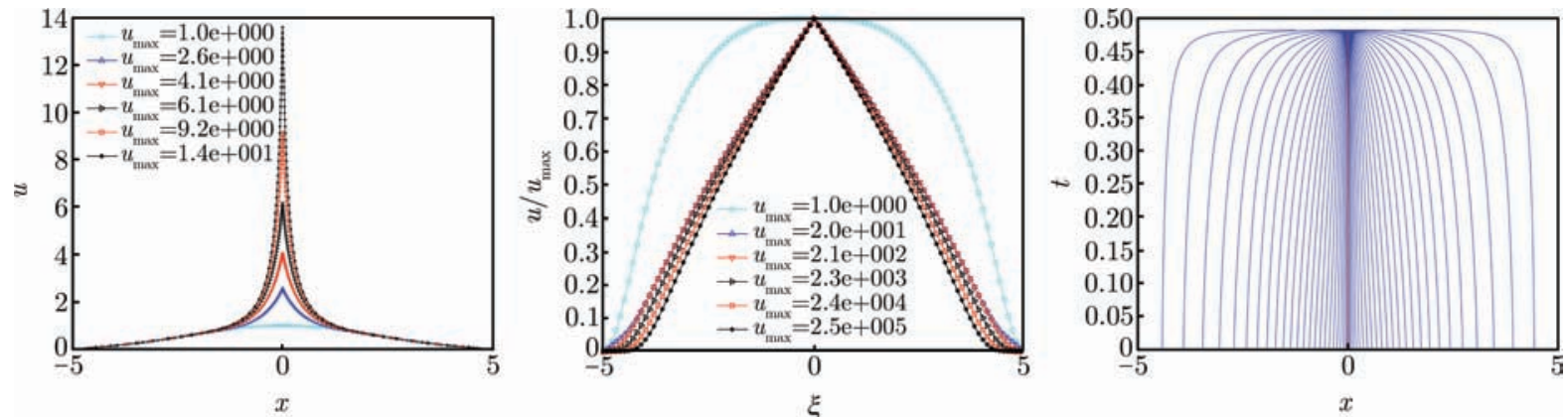

图 5 对于例 3 中 $k=0$ 情形, 从左到右分别为数值爆破解对物理变量和计算变量的图形以及移动网格轨迹图

这里 $\mu$ 是一个足够小的正常数.

实验中, 空间网格点数取为 $N=100 ;$ MMPDE6 方程中参数 $\tau$ 取为 $\tau=10^{-3},(60)$ 中 $\mu$ 取为 $\mu=10^{-3}$. 监视函数取为

$$
M(x, t)=\alpha u^{2}+(1-\alpha)\left(\left(x-x_{0}(t)\right)^{2}+\epsilon\right)^{-1 / 4},
$$

其中 $\alpha=0.5, \epsilon=10^{-5}$.

在这个例子中, $k=0$ 和 $k=2$ 两种情形将被测试. 计算结果为: 当 $k=0$ 时,数值计算得爆破时 刻为 $T=0.483364$, 此时计算解的最大值为 $u_{\max }=2.5091 \times 10^{5}$; 当 $k=2$ 时, 数值计算得爆破时刻为 $T=0.6032368$, 此时计算解的最大值为 $4.1698 \times 10^{6}$. 图 4 和图 5 给出了数值爆破解对物理变量和计 算变量的图形以及移动网格运动轨迹图. 


\section{6 结论及注记}

本文使用自适应网格重构算法求解带移动热源的反应扩散方程. 这种算法优点是易于实现. 多年 来该算法已被成功地应用于求解许多应用问题. 本文给出了这种算法的收玫性证明. 理论分析得到这 种算法的收玫阶与所使用的插值有直接关系: 使用线性插值时算法具有一阶收玫性, 使用二次插值时 算法具有二阶收玫性. 虽然我们的分析是针对线性方程进行的, 但实验结果显示理论分析结果对非线 性问题同样适用. 最后, 我们还用这类算法模拟了一类具有有限时间爆破解的非线性问题.

我们需要指出: 本文的收玫性分析使用了网格依赖 $L_{2}$ 范数. 自适应移动网格算法求解时间依赖 偏微分方程的 $L_{\infty}$ 范数收玫性分析始终还没得到解决. 其原因是算法的一致稳定性无法得到证明. 然 而使用等分步原理的移动网格算法求解椭圆问题的收玫理论已经被很好地解决了，参见代表性工作 [23-27].

最后, 我们指出使用 $L_{2}$ 投影移动有限元方法的收玫性分析已经被 Dupont 以及他的合作者们证 明 (例如: [28, 21, 29]), 但是使用一般插值移动有限元方法的收敛性分析一直是个挑战性问题. 另外, 本文的分析还暂时不能推广到高维问题. 总之, 移动网格方法的收玫性理论还远未被解决, 我们希望本 文以及现有文献的研究能对理解移动网格方法的整个理论有些启发作用.

致谢 本文第一作者对 Bob Russell 和 Weizhang Huang 关于第 3 和 4 节的讨论表示致谢, 并且所有作者衷心感谢 编委会和审稿专家们的宝贵意见。

\section{参考文献}

1 Kirk C M, Olmstead W E. Blow-up solutions of the two-dimensional heat equation due to a localized moving source. Anal Appl, 2005, 3: 1-16

2 Kirk C M, Olmstead W E. The influence of two moving heat sources on blow-up in a reactive-diffusive medium. Z Angew Math Phys, 2000, 51: 1-16

3 Kirk C M, Olmstead W E. Blow-up in a reactive-diffusive medium with a moving heat source. Z Angew Math Phys, 2002, 53: $147-159$

4 Bonnerot R, Jamet P. A second order finite element method for the one-dimensional Stefan problem. Inter J Numer Meth Eng, 1974, 8: 811-820

5 Jamet P. Stability and convergence of a generalized Crank-Nicolson scheme on a variable mesh for the heat equation. SIAM J Numer Anal, 1980, 17: 530-539

6 Ma J. Convergence analysis of moving Godunov methods for dynamical boundary layers. Comput Math Appl, 2010, 59: 80-93

7 Mackenzie J A, Mekwi W R. An analysis of stability and convergence of a finite-difference discretization of a model parabolic PDE in 1D using a moving mesh. IMA J Numer Anal, 2007, 27: 507-528

8 Ma J, Jiang Y, Xiang K. On a moving mesh method for solving partial integro-differential equations. J Comput Math, 2009, 27: 713-728

9 Mackenzie J A, Madzvamuse A. Analysis of stability and convergence of finite-difference methods for a reaction-diffusion problem on a one-dimensional growing domain. IMA J Numer Anal, doi: 10.1093/imanum/drp030

10 Blom J G, Sanz-Serna J M, Verwer J G. On simple moving grid methods for one-dimensional evolutionary partial differential equations. J Comput Phys, 1988, 74: 191-213

11 Li R, Tang T, Zhang P W. Moving mesh methods in multiple dimensions based on harmonic maps. J Comput Phys, 2001, 170: $562-588$

12 Li R, Tang T, Zhang P W. A moving mesh finite element algorithm for singular problems in two and three space dimensions. J Comput Phys, 2002, 177: 365-393

13 Zhang Z, Tang T. An adaptive mesh redistribution algorithm for convection-dominated problems. Comm Pure Appl Anal, 2002, 1: 341-357

14 Tang H, Tang T. Adaptive mesh methods for one- and two-dimensional hyperbolic conservation laws. SIAM J Numer Anal, 2003, 41: 487-515 
15 Tang H, Tang T, Zhang P W. An adaptive mesh redistribution method for nonlinear Hamilton-Jacobi equations in two- and three-dimensions. J Comput Phys, 2003, 188: 543-572

16 Di Y N, Li R, Tang T, et al. Moving mesh finite element methods for the incompressible Navier-Stokes equations. SIAM J Sci Comput, 2005, 26: 1036-1056

17 Zhang Z. Moving mesh method with conservative interpolation based on $L^{2}$-projection. Comm Comput Phys, 2006, 1: $930-944$

18 Budd C, Huang W, Russell R D. Adaptivity with moving grids. Acta Numer, 2009, 18: 111-241

19 Huang W, Russell R D. Adaptive Moving Mesh Methods. New York: Springer, 2011

20 Ma J, Jiang Y. Moving mesh methods for blowup in reaction-diffusion equations with traveling heat source. J Comput Phys, 2009, 228: 6977-6990

21 Bank R E, Santos R F. Analysis of some moving space-time finite element methods. SIAM J Numer Anal, 1993,30 $1-18$

22 Huang W, Ren Y, Russell R D. Moving mesh partial differential equations (MMPDEs) based upon the equidistribution principle. SIAM J Numer Anal, 1994, 31: 709-730

23 Qiu Y, Sloan D M, Tang T. Numerical solution of a singularly perturbed two-point boundary value problem using equidistribution: analysis of convergence. J Comput Appl Math, 2000, 116: 121-143

24 Kopteva N. Maximum norm a posteriori error estimates for a one-dimensional convection-diffusion problem. SIAM J Numer Anal, 2001, 39: 423-441

25 Kopteva N, Stynes M. A robust adaptive method for quasi-linear one-dimensional convection-diffusion problem. SIAM J Numer Anal, 2001, 39: 1446-1467

26 Chen L, Xu J. An optimal streamline diffusion finite element method for a singularly perturbed problem. In: Shi Z, Chen Z, Tang T, et al., eds. Recent Advances in Adaptive Computation. Contemp Math, 2005, 383: 191-201

27 Huang W. Convergence analysis of finite element solution of one-dimensional singularly perturbed differential equations on equidistributing meshes. Int J Numer Anal Modeling, 2005, 2: 57-74

28 Dupont T. Mesh modification for evolution equations. Math Comput, 1982, 39: 85-107

29 Dupont T, Liu Y. Symmetric error estimates for moving mesh Galerkin methods for advection-diffusion equations. SIAM J Numer Anal, 2002, 40: 914-927

\section{Analysis of an adaptive remeshing algorithm with interpolations for reaction- diffusion equations with traveling heat source}

\section{MA JingTang \& JIANG YingJun}

Abstract This paper studies an adaptive remeshing algorithm with interpolations for solving a kind of reactiondiffusion equations with moving source terms. The algorithm has two steps: The first step is to compute the spatial meshes at the new time level using the computational solutions at the old time level; The second step is to discretize the equations using finite difference methods on the spatial meshes at the new time level with the interpolations of solutions at the old time level as the initial values. For the time, a first-order convergence is obtained. For the space, the first-order convergence is proved for the algorithm with linear interpolations, and second-order convergence with quadratic interpolations. Numerical examples are carried out to confirm the theoretical findings.

Keywords: moving mesh methods, stability and convergence, reaction-diffusion equations

MSC(2000): $65 \mathrm{~N} 50,65 \mathrm{M} 50,35 \mathrm{~K} 55,35 \mathrm{~K} 57$

doi: $\quad 10.1360 / 012009-1050$ 